\title{
El sobrepeso y el mantenimiento de la pérdida de peso ${ }^{1}$
} Anne Mathews, Lauren Foster, and Wendy Dahl²

\section{¿Qué se considera sobrepeso?}

El sobrepeso y la obesidad, ambos grados de exceso de grasa corporal, son problemas de salud significativos. La grasa corporal se estima usando el índice de masa corporal"Body Mass Index" (BMI, por sus siglas en inglés), el cual se calcula usando la altura del individuo y el peso. El siguiente cuadro muestra las categorías que usamos para definir la grasa del cuerpo en los adultos usando el BMI.

\begin{tabular}{|l|c|}
\hline \multicolumn{1}{|c|}{ Estado } & BMI \\
\hline Peso bajo & $\leq 18.5$ \\
\hline Normal & $18.5-24.9$ \\
\hline Sobre peso & $25.0-29.9$ \\
\hline Obeso 1 & $30-34.9$ \\
\hline Obeso 2 & $35-39.9$ \\
\hline Extremadamente Obeso & $\geq 40$ \\
\hline
\end{tabular}

\section{¿Qué ocurre con los niños con sobrepeso?}

La tasa de crecimiento de los niños obesos es particularmente preocupante, considerando que muchos adolescentes obesos se convierten en adultos obesos (Goldhaber-Fiebert et al. 2012). Los adultos con sobrepeso y los obesos tienen un riesgo mucho más alto de contraer enfermedades crónicas incluyendo la diabetes, enfermedades cardiacas, cáncer de mama e hipertensión.

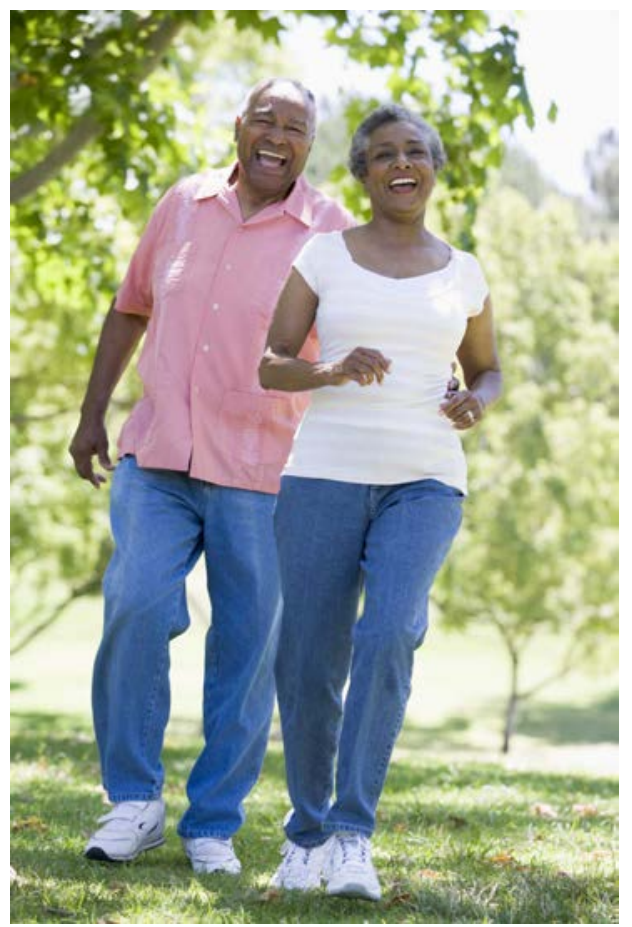

Figura 1. Hacer ejercicio y mantenerse activo le ayudará a aumentar su metabolismo a través del dia.

Credits: Thinkstock.com

\section{¿Qué podemos hacer para tomar acción?}

El primer paso es saber cuándo establecer un plan de manejo de peso para prevenir el aumento adicional de peso. La siguiente tabla muestra los tratamientos recomendados para personas de acuerdo a su BMI y de acuerdo a las condiciones concomitantes (enfermedades asociadas) como

1. Este documento, FSHN13-04s, es uno de una serie de publicaciones del Departmento de Alimentos Ciencias y Nutrición Humano, Servicio de Extensión Cooperativa de la Florida, Instituto de Alimentos y Ciencias Agrícolas, Universidad de la Florida. (UF/IFAS). Fecha de primera publicación: abril 2013. Visite nuestro sitio web EDIS en http://edis.ifas.ufl.edu.

2. Anne Mathews, profesora asistente; Lauren Foster, BS; Wendy Dahl, profesora asistente; Alimentos Ciencias y Nutrición Humano Departmento; Instituto de Alimentos y Ciencias Agrícolas; Universidad de Florida; Gainesville 32611. 
Copia de archivo: para las recomendaciones actuales, consulte http://edis.ifas.ufl.edu o su oficina de extensión local.

\begin{tabular}{|c|c|c|c|}
\hline & Dieta/Ejercicio & Terapia -fármacos & Cirugía Bariátrica \\
\hline Sobrepeso & $\mathrm{Si}$, si hay concomitantes & & \\
\hline Obeso I & $\mathrm{Si}$ & $\mathrm{Si}$, si hay concomitantes & $\mathrm{Si}$, si hay concomitantes \\
\hline Obeso 2 & $\mathrm{Si}$ & $\mathrm{Si}$ & $\mathrm{Si}$ \\
\hline Extremadamente obeso & $\mathrm{Si}$ & $\mathrm{Si}$ \\
\hline
\end{tabular}

la diabetes, la presión arterial alta y las enfermedades del corazón (Practical Guide: Identification, Evaluation, and Treatment of Overweight and Obesity in Adults 2000).

\section{¿Cómo bajar de peso?}

Para muchas personas, la pérdida de peso es una batalla crónica. Las dietas populares a menudo son poco exitosas porque no se pueden seguir de forma permanente. No hay una dieta mágica que le pueda hacer bajar de peso inmediatamente, pero a continuación se enumeran algunos buenos consejos para la pérdida de peso de una manera estable y de largo plazo.

1.Las porciones importan: La pérdida de peso y el mantenimiento del peso tiene que ver con el equilibrio y la moderación. Intente usar platos más pequeños y tazones que le ayuden a disminuir el tamaño de su porción ( Pedersen, Kang, and Kline 2007). Otro consejo es esperar al menos 10 minutos después de terminar de comer antes de volver por un segundo plato.

2. Sea activo: Hacer ejercicio y mantenerse activo le ayudará a aumentar su metabolismo durante todo el día.

3. Todo el mundo es diferente: Para que usted pueda mantener la pérdida de peso a largo plazo, debe estar familiarizado con su cuerpo y con sus hábitos alimenticios. Identifique qué situaciones o alimentos son particularmente tentadores para usted y trate de modificarlos, sin negárselos por completo

\section{Haga que la mitad de su plato tenga frutas y vegetales:} Seguir las pautas de MiPlato es una gran manera de reducir la grasa, las calorías y aumentar la densidad nutricional en sus comidas. Las frutas y los vegetales tienen una buena cantidad de fibra y de agua para ayudarle a mantenerse satisfecho.

\section{5. ¿Come usted cuando no tiene hambre? Algunas veces} esta bien, pero si es muy frecuente puede contribuir a ganar peso. Lleve un registro durante unos días de su nivel de hambre y saciedad antes y después de comer. Anote su estado de ánimo. Sea consciente qué el aburrimiento, la frustración, el estrés o la tristeza contribuyen a comer en exceso y es el primer paso para controlarse.
6. Pesarse regularmente: Los estudios demuestran que el pesarse regularmente (una vez a la semana) está asociado con una disminución del peso corporal y del mantenimiento del peso (Van Wormer et al. 2009)

7. No se salte las comidas: Distribuya el consumo total de calorías durante todo el día, con un consumo de 4 a 5 comidas/meriendas por día incluyendo el desayuno. El consumo de energía más temprano en el día puede ayudar a controlar mejor el hambre y comer menos durante el transcurso de día (de Castro 2004).

8. Rodéese de apoyo: Hable con su familia, amigos y compañeros de trabajo y dígales cómo pueden ayudarle, como no ofrecerle segundos platos y ofrecerle ensalada y fruta en vez de pizza y pastel en las fiestas.

9. Establezca metas para mantenerse motivado: Después de haber perdido peso y una vez que se terminen los cumplidos, puede sentirse como si no hay ningún incentivo para continuar con sus buenos hábitos. La clave es encontrar maneras de mantenerse en su meta de peso como también llegar a su peso ideal Regístrese para una caminata de la comunidad.Explore nuevas actividades que puede disfrutar como la jardinería, caminatas,tenis o aeróbicos en el agua, kayak o montar en bicicleta - o simplemente camine después de la cena como una rutina familiar en las tardes. Enfóquese en la calidad de su dieta y bríndese una recompensa, que no sea de alimentos, cuando alcance su meta del mantenimiento de peso.

\section{¿Por qué es tan difícil mantener el peso?}

El mantenimiento de la pérdida peso es complicado por muchos factores que lo hacen parecer una tarea imposible. Una pérdida de peso exitosa es a menudo seguida por un aumento de peso constante. Las personas que hacen dietas tienden a volver a los hábitos anteriores y disminuir las actividades diarias mientras pierden la "mentalidad de dieta". El perder peso también induce a los cambios hormonales o a reducir la masa corporal magra, lo que hace más difícil la pérdida de peso. Sin emabrgo, la actividad física puede ofrecer estos cambios. 


\section{¿La pérdida de peso a largo plazo es posible?}

¡Sí! Uno de cada 6 adultos han mantenido alrededor del $10 \%$ de su máximo peso durante al menos un año. ¿Cuál es su secreto? De acuerdo al Control de Registro Nacional del Peso- National Weight Control Registry (Wing and Hill 2001):

- $78 \%$ desayunan todos los días.

- $75 \%$ se pesan al menos una vez a la semana.

- $62 \%$ ven menos de 10 horas de televisión a la semana.

- 90\% hacen ejercicios en promedio alrededor de una hora.

\section{¿Dónde puedo obtener más información?}

El agente local de Ciencias de la Familia y el Consumidor (FCS) de la oficina de extensión de su condado puede tener más información escrita y clases de nutrición para que usted asista. También, un dietista registrado (RD) le puede proporcionar información confiable.

Más información nutricional confiable se puede encontrar en los siguientes sitios del Internet:

http://fycs.ifas.ufl.edu

http://solutionsforyourlife.ufl.edu

http://www.nutrition.gov

http://www.nal.usda.gov/fnic

\section{Referencias}

De Castro, J. M. 2004. The time of day of food intake influences overall intake in humans. J Nutr. 134: 104-111.

Goldhaber-Fiebert, J. D., R. E. Rubinfeld, J. Bhattacharya, T. N. Robinson, and P. H. Wise. 2012. The utility of childhood and adolescent obesity assessment in relation to adult health. Medical Decision Making. Stanford School of Medicine Cardiovascular Institute. [Epub ahead of print].

Pedersen, S. D., J. Kang, and G. A. Kline. 2007. Portion control plate for weight loss in obese patients with Type 2 diabetes mellitus. Arch Int Med. 167: 1277-83.

Practical Guide: Identification, Evaluation, and Treatment of Overweight and Obesity in Adults. 2000. National Heart, Lung, and Blood Institute, Publication No. 00-4084.

VanWormer, J. J., A. M. Martinez, B. C. Martinson, A. L. Crain, G. A. Benson, D. L. Cosentino, and N. P. Pronk.
2009. Self-weighing promotes weight loss for obese adults. Am J Prev Med. 36: 70-3.

Wing, R. R., and J. O. Hill. 2001. Successful weight loss maintenance. Annu Rev Nutr.; 21: 323-41. 